Western University Scholarship@Western

1970

\title{
On Production Functions and Elasticity of Substitution
}

K. R. Kadiyala

Follow this and additional works at: https://ir.lib.uwo.ca/economicsresrpt

Part of the Economics Commons

Citation of this paper:

Kadiyala, K. R.. "On Production Functions and Elasticity of Substitution." Department of Economics Research Reports, 7024. London, ON: Department of Economics, University of Western Ontario (1970). 
Echountes.

SEP 11970

\author{
RESEARCH REPORT 7024 \\ ON PRODUCTION FUNCTIONS \\ AND ELASTICITY OF SUBSTITUTION \\ by
}

K. R. Kadiyala

September, 1970 
ON PRODUCTION FUNCTION:S

AND EIASTICITY OF SUBSTITUTION

by

K. R. Kadiyala

1. Introduction

It is not an overstatement to say that research in empirical economics has tremendously increased both in quality and quantity ever since Cobb and Douglas proposed the Cobb-Douglas production function for finding relationships among economic variables. Two important reasons for this are the simplicity of the Cobb-Douglas production function from estimation point of view and that it explains most economic data quite adequately. Even though the Cobb-Douglas production function describes most economic data to a very good accuracy, it has some serious drawbacks. The main ones being that it requires all inputs to be positively employed and that it has a unitary elasticity of substitution for all levels of factors. The generalized constant elasticity of substitution (CES) production functions introduced by Arrow, Chenay, Minhas and Solow (ACMS) [2] took care of the first of the above criticisms. But if the elasticity of substitution were to be an index for judging a production function as a means of explaining relationships among economic variables the CIS production function has the same drawback as the Cobb-Douglas prodiction function. Intuitively speaking, one would expect thet as one moves along a given isoquant the elasticity of substitution to increase (decreaie) as the relevant input ratio goes to infirity as well as to zero. As Ailen [I, p. 342] puts 
it "the larger is the value of $\sigma,{ }^{1}$ the flatter is the constant product curve and the more slowly does the marginal rate of substitution increase as $B$ is substituted for A. The magnitude of $\sigma$ is thus an indication of the ease with which product can be maintained by substituting B for A." Obviously the CES (and hence the Cobb-Douglas) production functions do not possess this desirable property. Recently some attempts have been made to find production functions which could meet this criticism. In this connection special reference ${ }^{2}$ should be made to the works of Iu and Fletcher [3], Revanker [4] and Sato and Hoffman [6]. The general approach of these studies has been to assume that the elasticity of substitution is a linear function of the ratio of the two inputs and integrate the resulting differential equation to arrive at the implied production function. The resulting production functions, therefore do possess the property of exhibiting variable elasticity of substitution and, in a way, are a generalization of the CES production functions. But by the very nature of the assumptions made these production functions have the property that the elasticity of substitution is either a monotone increasing function of the input ratio or a monotone decreasing function of the input ratio. This means that the elasticity of substitution either reaches a maximum or a minimum as the input ratio increases (decreases). This property is against one's intuition because the elasticity of substitution of the input $x_{1}$ for $x_{2}$ is the same as the elasticity of substitution of $x_{2}$ for $x_{1}$. Therefore one would expect the

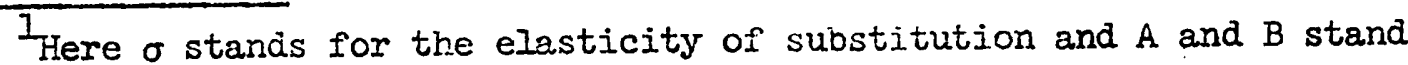
for the two inputs.

${ }^{2}$ Here no attempt is made to give a complete list of studies that have been done on this topic nor is any attention paid to the chronology. 
elasticity of substitution to increase (decrease) as the input ratio increases as well as it decreases when the input ratio gets sufficiently small. In this paper a simple production function is presented. This production function includes as special cases the CES production function, the Lu-Fletcher [3] production function, the Revanker [4] and Sato-Hoffman [6] production functions and also is quite simple from estimation point of view. The production function is presented in section two. Section three presents some of the properties of the production function. Some generalizations and conclusions are presented in Section 4.

\section{The Production Function}

We assume the output $Y$ is a function two ${ }^{3}$ inputs, $X_{1}$ and $X_{2}$ the production function that is being proposed is given by

$Y=F\left(X_{1}, X_{2}\right)=E(t)\left(w_{11} X_{1}^{2 p}+2 w_{12} X_{1}^{p 1} x_{2}^{p^{2}}+w_{22} x_{2}^{2 p}\right)^{1 / 2 p}$.

Here $E(t)$ stands (using ACMS terminology) for the efficiency parameter which also absorbs the neutral technical progress. $w_{11}, w_{12}$ and $w_{22}$ are assumed to be non-negative. If the technical progress is neutral they will be independent of time, otherwise they are also functions of time (i.e., we have some kind of chronological ordering of the production process). We assume, without loss of generality, that $w_{11}+2 w_{12}+w_{22}=1$. It is clear that $F$ is homogeneous of degree one ${ }^{4}$

3 The case of more than two inputs is brieriy aiscussed in Section 4.

4 Increasing (decreasing) returns to wcale can be coyered by trivially extending (I) to $F\left(X_{1}, X_{2}\right)=E(t)\left(w_{11} X_{1}{ }^{2} p+2 w_{12} X_{1} \rho 1 X_{2} \rho^{2}+\omega_{22} X_{2}^{2 p}\right) v / 2 p$ where $\nu>1(0<\nu<1)$. 
in the inputs $x_{1}$ and $x_{2}$ (i.e., constant returns to scale). It is assumed that $p_{1}+p_{2}=2 p$ and that $p_{1}$ and $p_{2}$ have the same sign as $p$. This assumption is necessary to insure that the marginal products are non-negative. This production function is a generalization of the CES production function, the Iu-Fletcher production function and the Revanker and Sato-Hoffman production function. For, by letting $\omega_{12}=0 ; \omega_{22}=0$; and $\omega_{11}=0$ (I) reduces to the CES, the Iu-FIetcher and the Revanker-Sato-Hoffman production functions, respectively. We will not pursue the production function (I) in that form. We will make a slight simplifying assumption and then study its properties. Therefore, from here on we will assume that $p_{1}=p_{2}=0$. This assumption does restrict the form (I) to the degree that it has one parameter less. But from an empirical research worker's point of view it is much more easily adoptable and still maintains its essential characteristics. With this assumption (1) can be written as

$Y=F\left(X_{1}, X_{2}\right)=E(t)\left(\omega_{11} X_{1}^{2 p}+2 \omega_{12} X_{1}^{p} X_{2}^{p}+\omega_{22} X_{2}^{2 p}\right)^{1 / 2 p}$

It is clear that (2) and CES production functions are the same in the following sense. The CES production functions and (2) are homogeneous of degree $1 / 2$ in the variables $x_{i j}=x_{i} x_{j} ; 1, j=1,2$. The only difference being that the CES production functions assume the cross term, $\omega_{12}$ to be zero. The parameter $\omega_{12}$ can be interpretted as a reaction parameter between the inputs $X_{1}$ and $X_{2}$. Because of this slight modification a great deal of variability is achieved by using the form (2)

It should be noted that this is only one of several ways of looking at (I) as a generalization of the CES production function. 
as opposed to the CES production function or its generalizations [2, 4, 6] as will be seen from the following discussion.

\section{Properties of the Production \\ Function}

The production function (2) is homogeneous of degree one in the inputs $X_{1}$ and $X_{2}$ and the variables $Y^{l / \rho} ; X_{1}^{\rho}$ and $X_{2}^{1 / \rho}$ are related by a quadratic form. More specifically (2) can also be written as

$$
\left(\begin{array}{llll}
y & x_{2}
\end{array}\right) A\left(\begin{array}{lll}
y & x_{2}
\end{array}\right)^{\prime}=0
$$

where $A$ is the $3 \times 3$ matrix given by

$$
A=\begin{array}{ccc}
-1 & 0 & 0 \\
0 & E^{2 p} \omega_{11} & E^{2 p} \omega_{12} \\
0 & E^{2 p} \omega_{12} & E^{2 p} \omega_{22}
\end{array}
$$

and $y=y^{1 / p}, x_{1}=x_{1}^{1 / p}$ and $x_{2}=x_{2}^{1 / p}$. It can be quickly verified that the marginal products are non-negative. In the admissable space of the parameters the second direct partial derivatives are negative and the second cross partial derivative is positive as will be seen below (equation (5)). The factor elasticity of substitution, $\sigma$ is given by

$$
\sigma(x)=\frac{1}{1-\rho+R}
$$

where

$$
R=\frac{-\rho\left(w_{11} \omega_{22}-\omega_{12}^{2}\right)}{\left(w_{11} x^{-\rho}+\omega_{12}\right)\left(w_{12}+\omega_{22} x^{\rho}\right)}
$$

and $x=x_{2} / x_{1} \cdot$ Since 


$$
\frac{\partial R}{\partial x}=\frac{\rho^{2}\left(\omega_{11} \omega_{22}-\omega_{12}{ }^{2}\right) \omega_{12} x^{-\rho-1}\left(\omega_{22} x^{2 \rho}-\omega_{11}\right)}{\left(\omega_{11} x^{-\rho}+\omega_{12}\right)^{2}\left(\omega_{12}+\omega_{22} x^{\rho}\right)^{2}}
$$

assuming ${ }^{6} \omega_{11} \omega_{22}-\omega_{12}^{2}>0, R$ increases (decreases) with $x$ as long as $x \geq\left(\omega_{11} / \omega_{22}\right)^{I / 2 \rho},\left(x<\left(\omega_{11} / \omega_{22}\right)^{I / 2 \rho}\right.$ and increases for $x>\left(\omega_{11} / \omega_{22}\right)^{I / 2 \rho}$. When $\rho<0, \sigma$ decreases up to the point $x=\left(\omega_{12} / \omega_{22}\right)^{1 / 2 \rho}$ and increases for $x>\left(\omega_{17} / \omega_{22}\right)^{I / 2 \rho}$. Therefore when $\rho>0$ the maximum elasticity is attained at $x=\left(\omega_{11} / \omega_{22}\right)^{1 / 2 \rho}$ and equals $\sigma_{U}+$ where

$$
\sigma_{\mathrm{U}}+=\frac{1}{1-\rho-\rho \frac{\omega_{11} \omega_{22}-\omega_{12}^{2}}{\left(\omega_{11}^{1 / 2} \omega_{22}^{1 / 2}+\omega_{12}\right)^{2}}}
$$

and the minimum elasticity of substitution is attained as $\mathrm{x}$ tends to its two end points, 0 and $\infty$ and equals $\sigma_{\mathrm{L}}{ }^{+}$

where

$$
\sigma_{I}^{+}=\frac{I}{1-\rho}
$$

This implies that $\rho \leq \frac{\left(\omega_{11}^{1 / 2} \omega_{22}^{1 / 2}+\omega_{12}\right)^{2}}{2\left(\omega_{11} \omega_{22}+\omega_{11}^{1 / 2} \omega_{22}^{1 / 2} \omega_{12}\right)}$.

When $\rho<0$ the maximum elasticity is attained as $x$ approaches 0 and $\infty$ and equals $\sigma_{U}-$ where

$$
\sigma_{U}^{-}=I / I-p
$$

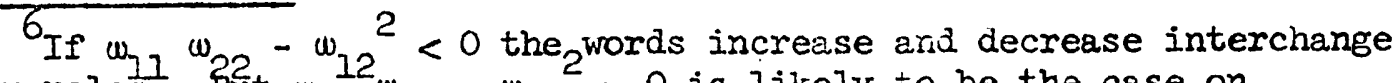
their roles. But $\omega_{11} w_{22}-w_{12}>0$ is iikely to be the case on intuitive grounds.
} 
The minimum elasticity is attained at $x=\left(\omega_{11} / \omega_{22}\right)^{1 / 2 p}$ and equals $\sigma_{L}^{-}$, where

$$
\sigma_{I}^{-}=\frac{1}{1-\rho-\rho \frac{\omega_{11} \omega_{22}-\omega_{12}^{2}}{\left(\omega_{11}{ }^{1 / 2} \omega_{22}^{1 / 2}+\omega_{12}\right)^{2}}}
$$

It can be easily verified that (2) tends to (i) a Cobb-Douglas production function as $\rho \rightarrow O$ (ii) a Ieontief production function as $\rho \rightarrow-\infty$ and (iii) a linear ${ }^{7}$ production function as $w_{12} \rightarrow 0$ and $p \rightarrow 1 / 2$. The ratio of the factor earnings is given by $X_{1} F_{1} / X_{2} F_{2}$ and equals

$$
\begin{array}{r}
\frac{x_{1} F_{1}}{x_{2} F_{2}}=\frac{w_{11}+\left(1-w_{11}-w_{22}\right) x^{\rho}}{\left(1-w_{11}-w_{22}\right) x^{\rho}+w_{22} x^{2 \rho}} \\
\text { 4. Summary and Conclusions }
\end{array}
$$

In this paper we have extended the CES production functions to a class of production functions which are homogeneous and which possess the property that the elasticity of substitution varies with the input ratio as one moves along an isoquant and which is symmetric with respect to the two end points, 0 and $\infty$ of the input ratio. Even though the discussion of the paper has confined to two inputs the generalization to more than two inputs is immediate. One generalization to more than two inputs is clear. For, (2) can be written, when $k$ inputs are employed as

$$
Y=F\left(X_{1}, \ldots, X_{k}\right)=E(t)\left(\sum_{i=1}^{k} \omega_{i i} x_{i}^{2} p+2 \sum_{i<j} \omega_{i j} X_{i}^{p} X_{j}^{p}\right)^{1 / 2 p}
$$

\footnotetext{
7 of course, this is only one of several ways the way (2) approaches this form.
} 


\section{$-8-$}

where $\omega_{i j} \geq 0, i, j=1, \ldots, k$ and $\sum_{i=1}^{k} \omega_{i i}+\sum_{i<j} \omega_{i j}=1$. One can also compute the Allen or Uzawa-McFadden partial elasticities of substitution. But, when more than two inputs are involved both the Allen elasticities of substitution and the Uzawa-McFadden elasticities of substitution will, in general, involve all the input ratios and they will not be as simple as (5). If there are reasons to believe that there are internal economics of scale (or the economies of scale are a function of the output) one can make a transformation of (2) like, say Revanker and Zellner [5] to get a production function which exhibits internal economies and still maintains the properties $(5),(6),(7),(8)$ and (9). To characterize the production function (2), in general, is not possible. For, for every $\sigma(x)$ there corresponds a production function and in most cases it is not possible to solve for the specific form of the production function for a given elasticity of substitution function $\sigma(x)$. The next step is to see how good the production function explains relationships among economic variables, particularly in those cases where the CES and Cobb-Douglas production functions fail to give a reasonable fit. It should be noted that the number of parameters in (2) exceed that of the CES production function just by one. Therefore any substantial gain that is achieved by ritting (2) rather than the CES or the Cobb-Douglas production functions could be attributed to the form of the production function and not due to the many additional parameters introduced. The author is presently doing a study comparing these three production functions using data for which the correlation coefficient obtained by using CES or Cobb-Douglas production Iunction is relatively low. These results will be reported in a later study. 
[I] Allen, R. G. D., Mathematical Analysis For Economists (Iondon: Macmilian Company, 1938).

[2] Arrow, K. J., H. B. Chenery, B. S. Minhas, and R. M. Solow, "Capital-Labor Substitution and Economic Efficiency," The Review of Economics and Statistics, No. 3 (August 1961) pp. 225-250.

[3] Iu, Yao-Chi and Fletcher, I. B., "A Generalization of the CES Production Function," The Review of Economics and Statistics (November 1968).

[4] Revanker, N. S., "The Constant and Variable Elasticity of Substitution Production Functions: A Comparative Study in U. S. Manufacturing Industries," University of Wisconsin Systems Formulation, Methodology and Public Workshop Paper,6616, (Unpublished) 1966.

[5] Zellner, A. and Revanker, N. S., "Generalized Production Functions," Review of Economic Studies (April 1969).

[6] Sato, Rand Hoffman, R. F., "Production Functions with Variable Elasticity of Factor Substitution: Some Analysis and Testing," The Review of Economics and Statistics (November 1968). 